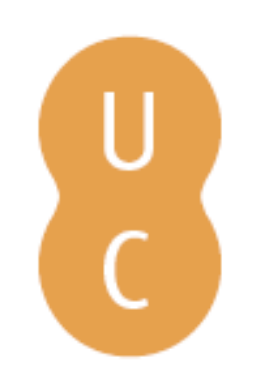

\title{
pompalina
}

\section{Geração automática de orto-mosaicos de fotos aéreas de arquivo do Concelho de Coimbra}

Autor(es): $\quad$ Gonçalves, José Alberto; Manta, Virgínia; Carvalho, Mário

Publicado por: Imprensa da Universidade de Coimbra

URL

persistente: URI:http://hdl.handle.net/10316.2/37097

DOI: $\quad$ DOI:http://dx.doi.org/10.14195/978-989-26-0983-6_44

Accessed : $\quad$ 26-Apr-2023 12:51:18

A navegação consulta e descarregamento dos títulos inseridos nas Bibliotecas Digitais UC Digitalis, UC Pombalina e UC Impactum, pressupõem a aceitação plena e sem reservas dos Termos e Condições de Uso destas Bibliotecas Digitais, disponíveis em https://digitalis.uc.pt/pt-pt/termos.

Conforme exposto nos referidos Termos e Condições de Uso, o descarregamento de títulos de acesso restrito requer uma licença válida de autorização devendo o utilizador aceder ao(s) documento(s) a partir de um endereço de IP da instituição detentora da supramencionada licença.

Ao utilizador é apenas permitido o descarregamento para uso pessoal, pelo que o emprego do(s) título(s) descarregado(s) para outro fim, designadamente comercial, carece de autorização do respetivo autor ou editor da obra.

Na medida em que todas as obras da UC Digitalis se encontram protegidas pelo Código do Direito de Autor e Direitos Conexos e demais legislação aplicável, toda a cópia, parcial ou total, deste documento, nos casos em que é legalmente admitida, deverá conter ou fazer-se acompanhar por este aviso.

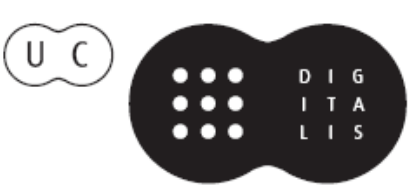




\section{$\forall$ \\ TAS DAS I JORNADAS LUSÓFONAS DE CIÊNCIAS E TECNOLOGIAS DE INFORMAÇÃO GEOGRÁFICA}

Editores

José Gomes dos Santos

Cidália Fonte

Rui Ferreira de Figueiredo

Alberto Cardoso

Gil Gonçalves

José Paulo Almeida

Sara Baptista 


\title{
A RTIGO 44
}

\section{GERAÇÃo AUTOMÁtica de ORTO-mOSAICOS DE FOTOS AÉrEAS DE ARQUiVo do CONCELHO DE COIMBRA}

\author{
GONÇALVES, José Alberto ${ }^{1}$; MANTA, Virgínia ${ }^{2}$; CARVALHO, Mário ${ }^{2}$
}

\footnotetext{
${ }^{1}$ Faculdade de Ciências da Universidade do Porto; Departamento de Geociências, Ambiente e Ordenamento do Território; Rua do Campo Alegre, 687, 4169-007 Porto, Portugal; Tel.: 22-0402000; Fax: 22-0402009; e-mail: jagoncalfc.up.pt

2 Câmara Municipal de Coimbra; Praça 8 de Maio - 3000-300 Coimbra; Tel.: 239828 078; Fax: 239820 114; e-mail: virginia.manta@cm-coimbra.pt, mario.carvalho@cm-coimbra.pt
}

\section{RESUMO}

A utilização de Sistemas de informação geográfica na gestão municipal requer muitas vezes informação antiga para confronto com a atual, sendo de particular interesse as fotografias aéreas de arquivo. No caso do concelho de Coimbra é frequentemente usada uma cobertura aérea de 1969, que, apesar de já digitalizada, não é eficazmente aproveitada por não estar georreferenciada. Este artigo descreve um procedimento essencialmente automático de georreferenciação com ortorretificação, e montagem de um mosaico único, realizado sobre as cerca de 750 fotos desta cobertura. Recorreu-se a uma metodologia comum à usada no processamento fotogramétrico de imagens de veículos aéreos não tripulados. Baseia-se em algoritmos recentes da visão computacional, que permitem a deteção de grande número de pontos conjugados entre as diferentes imagens, seguido de um ajuste de feixes com um número de pontos de controlo relativamente reduzido. Feita a extração automática de um modelo digital de superfície é composto o orto-mosaico final. Este produto foi validado de acordo com as necessidades de rigor do SIG municipal, sendo pontualmente editado e corrigido no sentido de criar um produto mais apelativo para os utilizadores. 
Atas das I Jornadas Lusófonas de Ciências e Tecnologias de Informação Geográfica, Sessão 10, Artigo 44 Geração automática de orto-mosaicos de fotos aéreas de arquivo do concelbo de Coimbra

José Goncalves, Virgínia Manta \& Mário Carvalho

\title{
PALAVRAS-CHAVE
}

Fotografia aérea, Triangulação aérea, Orientação automática, Orto-mosaico, MDS.

\section{AUTOMATIC GENERATION OF ORTHO-MOSAICS FROM \\ ARCHIVED AERIAL PHOTOGRAPHS OF THE \\ COIMBRA MUNICIPALITY}

\begin{abstract}
The use of a geographical information system in local authorities frequently requires historical data in order to analyse and compare with the present day situation. Archives of historical aerial photography are of particular interest to be used as a GIS layer. In the case of the Coimbra Municipality an aerial coverage dating from 1969 is frequently used. Although being digitised, it is not efficiently used since it is not georeferenced. This paper describes an essentially automatic process of ortho-rectification, georeferencing and mosaic composition from a set of more than 750 photographs of this coverage. The methodology is the same as the one used in the photogrammetric processing of imagery acquired by unmanned aerial vehicles. It is based on recent computer vision algorithms, which allow for the detection of many conjugate points, followed by a bundle adjustment, with a relatively small number of ground control points. Once an automatic digital surface model was extracted all images were ortho-rectified and a mosaic was built. This product was validated according to the positional accuracy rules of the municipal GIS. In some areas the orho-image was edited in order to create a more appealing product for the users.
\end{abstract}

\section{KEYWORDS}

Aerial photography, Aerial triangulation, Automatic orientation, Orthomosaic, DSM.

\section{INTRODUÇÃO}

A utilização de Sistemas de informação geográfica (SIG) requer informação atualizada, que represente a situação presente da região de estudo. Contudo, em muitas atividades, como é o caso da gestão municipal, a disponibilidade de informação histórica é também de grande interesse pela possibilidade de conhecer situações passadas de ocupação do território. Um exemplo típico prende-se com questões de licenciamento: sendo necessário apresentar a licença de utilização para diversos atos legais, no caso de não existir processo de licenciamento é necessário apresentar 
comprovativo em como o edifício foi construído antes de 7 de agosto de 1951, se se situar no perímetro urbano da cidade de Coimbra ou antes de 12 de maio de 1962 se se situar no exterior do perímetro urbano.

A cartografia em papel, de uma dada época, pode facilmente ser digitalizada e georreferenciada, sendo tratada no SIG como uma camada raster representativa de uma época. Esse procedimento é feito rotineiramente, não apresentando dificuldades de maior. Frequentemente estão também disponíveis coberturas de fotografias aéreas que são igualmente fornecedoras de informação histórica de grande interesse, contudo o processo de georreferenciação é menos simples, exigindo procedimentos do âmbito da Fotogrametria, não dominados pela maioria dos utilizadores de informação geográfica. Além disso, esses procedimentos envolvem, na sua forma mais comum, trabalho demorado e custos significativos, que diminuem o interesse da sua execução.

No caso do Concelho de Coimbra encontram-se disponíveis várias coberturas aéreas, sendo uma delas uma das primeiras efetuadas em Portugal, para fins de produção cartográfica. Trata-se de um voo de 1932, realizado para a produção da planta de escala 1:1000 de Coimbra (MANTA et al., 2011). Esta planta foi também digitalizada e georreferenciada, sendo uma camada frequentemente utilizada no SIG municipal.

A partir dos anos 40 foram feitas regularmente coberturas aéreas do país, sendo algumas delas fornecidas pelas instituições detentoras. É o caso da cobertura de 1947, efetuada pela Força Aérea Inglesa, e a de 1958, pela Força Aérea Americana (REDWEIK, 2010). As duas são fornecidas pelo Instituto Geográfico do Exército, digitalizadas, mas não georreferenciadas.

Outras coberturas realizadas em Portugal são também de grande interesse. No caso de Coimbra, é comummente usada uma cobertura de 1969, realizada pela Força Aérea Portuguesa, que por ser de grande escala (escala média 1:6500) apresenta um detalhe adequado para a deteção de pormenor necessário para o SIG municipal.

Esta cobertura foi digitalizada, sendo acessível aos utilizadores do SIG como imagens não georreferenciadas. A única referência espacial consiste numa localização aproximada dos centros de projeção das diferentes 
fotos. Para uma dada região em análise é identificada a foto que a cobre, podendo ser visualizada mas sem georreferenciação, limitando-se o seu uso a uma fotointerpretação com localização aproximada. Seria de todo o interesse proceder à ortorretificação desta importante informação, de forma a poder ser visualizada corretamente sobreposta à informação atual.

Numa primeira abordagem considerou-se a possibilidade da sua ortorretificação pelos processos convencionais da fotogrametria. Trabalhos recentes desenvolvidos em Portugal sobre coberturas das décadas de 1940 e 1950 (REDWEIK et al., 2010) permitiram concluir que esta abordagem é possível de ser realizada. Contudo algumas dificuldades podiam ser de imediato identificadas. Em primeiro lugar não eram conhecidos parâmetros de calibração da câmara, necessários à execução do trabalho. Seria também necessário utilizar um modelo digital do terreno atual, o que poderia levantar problemas nas zonas que sofreram alterações topográficas significativas. Apesar de estes problemas poderem ser contornáveis ou não terem um impacto significativo, a principal dificuldade estava nos custos envolvidos, já que a ortorretificação só poderia ser realizada por uma empresa da área da Fotogrametria, com meios técnicos dessa especialidade, e com um significativo volume de trabalho de operadores especializados para tratar as cerca de 750 fotos deste voo. Apesar da importância deste voo como camada de dados para o SIG, os custos envolvidos não justificavam esta execução.

Dado que existem atualmente no contexto da Fotogrametria abordagens mais automáticas, em particular para processamentos muito mais expeditos, como é o caso do processamento de imagens adquiridas com VANTS (EISENBEISS, 2011, HAALA N., 2009, HAALA \& ROTHERMEL, 2012), analisou-se a possibilidade de recorrer a alternativas de software deste tipo para resolver o problema em causa. Foi utilizado o programa Agisoft Photoscan para a geração de um orto-mosaico, o que foi possível obter de uma forma essencialmente automática. Contudo foi necessário trabalho preparatório para que fosse possível aplicar esta abordagem. Descreve-se no restante deste artigo a metodologia seguida e os resultados alcançados. 


\section{CRIAÇÃO DE ORTO-MOSAICOS COM IMAGENS DE VANT}

O método seguido pelos programas de processamento de imagens de VANT baseia-se no conceito "Structure from Motion" (SFM). O programa utilizado, conforme referido, foi o Agisoft Photoscan (AGISOFT, 2012) cuja sequência de procedimentos é descrita a seguir. A figura 1 representa um fluxograma com os passos desse processamento. Os vários passos estão descritos abaixo em detalhe.

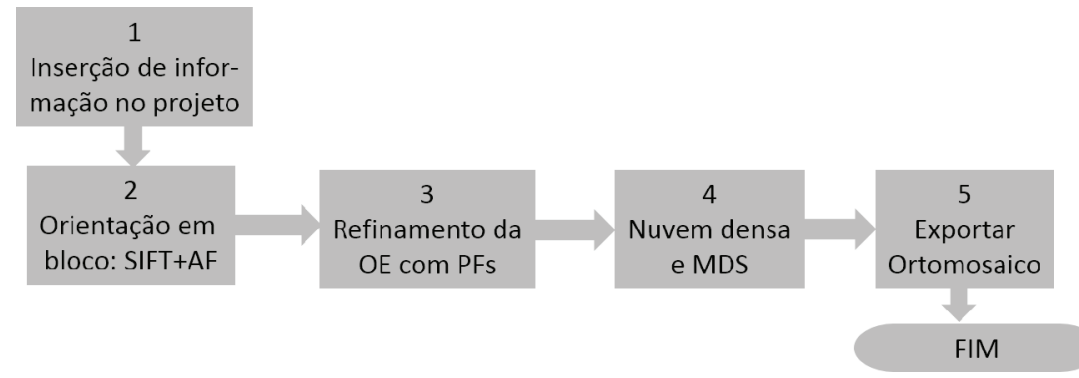

Figura 1 - Fluxograma das operações a realizar, com o programa Photoscan, na criação de orto-mosaicos adquiridos com um VANT

1. Inserção de informação no projeto:

Nesta fase são indicadas as fotos a processar. É feita a leitura da distância focal e dimensão do pixel (valores nominais da câmara), para orientação interna aproximada. Assume-se o ponto principal no centro da imagem e a não existência de deformações radial ou tangencial. Nesta fase é também feita a leitura da posição aproximada dos centros de projeção, resultantes do posicionamento GPS efetuado pelo equipamento de navegação. Normalmente estes dados são armazenados no cabeçalho das imagens JPEG, de acordo com o standard EXIF (HARVEY, 2014).

\section{Orientação em bloco: SIFT+AF}

Esta fase é designada no programa Photoscan pela expressão "alinhamento das imagens". Trata-se de uma expressão oriunda da comunidade da visão computacional, que corresponde à orientação relativa de pares de imagem, num processamento único, em bloco, recorrendo a pontos conjugados em grande número (centenas ou milhares por foto) identificados por métodos automáticos como o SIFT (Scale Invariant Feature Transform, LOWE, 2004). Segue-se o ajuste de feixes (AF), que na ausência 
de qualquer controlo terrestre seria efetuada num sistema de coordenadas arbitrário, mas que no caso presente, com o conhecimento das posições aproximadas dos centros de projeção, permite a obtenção de orientações externas aproximadas. Dada a elevada redundância de pontos conjugados é também possível incorporar neste processo uma auto-calibração da câmara, em que são refinados parâmetros de orientação interna (distância focal, posição do ponto principal e coeficientes do polinómio da distorção radial). Para os pontos conjugados são calculadas coordenadas 3D aproximadas, dando origem a uma nuvem esparsa.

3. Inserção de pontos de controlo e refinamento da orientação

É inserida uma lista de pontos de controlo (pontos fotogramétricos, PF), com coordenadas terreno conhecidas. Com a orientação externa (OE) aproximada esses pontos são localizados nas imagens, também de forma aproximada, devendo o utilizador corrigir a sua posição. Este é um procedimento manual, mas ainda assim fortemente suportado por uma previsão bastante boa da posição dos pontos. É repetido o ajuste de feixes, cujo resultado ficará agora corretamente georreferenciado no sistema de coordenadas em que se encontram os PF. Resultam também os erros médios quadráticos nos pontos de controlo e nos pontos de ligação, que servem para a deteção de erros grosseiros e avaliar globalmente a qualidade do processo. As coordenadas dos pontos da nuvem esparsa são também recalculadas.

4. Densificação da nuvem de pontos e criação de um modelo digital de superfície

A nuvem de pontos esparsa é densificada recorrendo a métodos de multi vision stereo. O programa Photoscan usa uma estratégia própria mas baseada na abordagem "Patch-based multi-view Stereo" (FURUKAWA e PONCE, 2010). O utilizador escolhe a densidade através de designações: "alta", correspondendo a 1 ponto em cada 2x2 pixels da imagem original, "média" para 1 ponto em cada 4x4 e "baixa" para 1 ponto em cada 8x8). Daí são calculadas coordenadas terreno 3D, a partir de todas as imagens, dando assim origem à nuvem de pontos densa. Essa nuvem 
densa é triangulada gerando um modelo digital de superfície (MDS) de toda a área coberta por 2 ou mais fotos, na forma de rede irregular de triângulos.

5. Exportação do orto-mosaico (e do MDS)

Cada uma das fotos é ortorretificada e é criado um mosaico de todas elas para toda a região, ou sub-região especificada pelo utilizador. Dado que a ortorretificação ocorre com um MDS, tratar-se-á de um "orto verdadeiro", que ortorretifica edifícios e vegetação, e que obriga a tratar cuidadosamente as oclusões nas diferentes fotos (GONÇALVES, et al., 2002), sendo uma operação relativamente demorada. Este procedimento é completamente automático, não sendo possível, pelo menos de forma simples, a ortorretificação com outro modelo que não o extraído. As linhas de corte, onde se dá uma transição suave entre as imagens, são também escolhidas automaticamente pelo programa. Nesta fase pode ser exportado também o MDS, na forma de grelha.

Este conjunto de procedimentos sequencial é facilmente seguido, mesmo por operadores não especialistas, sendo relativamente fácil produzir resultados de qualidade, com imagens de VANT. No caso presente, tratando-se de uma situação não standard, houve que fazer um conjunto de adaptações que estão descritas na secção seguinte.

\section{AplicaÇão da metodologia}

As fotos foram digitalizadas na CMC com uma resolução baixa, do ponto de vista dos padrões habituais da Fotogrametria, concretamente 200 dpi, ou seja um pixel de cerca de $127 \mu \mathrm{m}$. Na escala média das fotos trata-se de uma resolução no terreno de $0.8 \mathrm{~m}$, o que para as aplicações que se pretendiam no SIG municipal era suficiente. Por outro lado, o volume de dados correspondente às 754 fotos foi aceitável para um processamento num bloco único. 


\subsection{Preparação dos dados}

As imagens adquiridas por câmaras digitais, como é habitual num VANT, têm todas o mesmo tamanho e a mesmo posicionamento dos pixéis relativamente ao sistema fotográfico. Isso não acontece com as fotos digitalizadas em scanner, sendo necessário recorrer a marcas fiduciais para o relacionamento com o sistema fotográfico. Para usar o programa Agisoft Photoscan torna-se necessário retificar as imagens, de forma que todas tenham o mesmo tamanho e a mesma localização no sistema de coordenadas fotográficas.

As fotos foram retificadas, recorrendo às marcas fiduciais, através de uma transformação afim. Dado que não havia qualquer tipo de informação relativa a calibração de marcas fiduciais, utilizaram-se os valores nominais das câmaras Wild. Sendo as distâncias nominais entre marcas de $212 \mathrm{~mm}$, admitindo simetria em relação ao centro, as coordenadas das marcas fiduciais são, então, as da Tabela 1 .

Tabela 1 - coordenadas fotográficas consideradas para as marcas fiduciais

\begin{tabular}{|c|c|c|c|}
\hline Marca & Localização & $\mathbf{x}(\mathbf{m m})$ & $\mathbf{y}(\mathbf{m m})$ \\
\hline 1 & Canto superior esquerdo & -106.000 & +106.000 \\
\hline 2 & Canto superior direito & +106.000 & +106.000 \\
\hline 3 & Canto inferior direito & +106.000 & -106.000 \\
\hline 4 & Canto superior esquerdo & -106.000 & -106.000 \\
\hline
\end{tabular}

Este procedimento pode ser feito manualmente em qualquer programa que permita correções geométricas de imagens, como é o caso dos programas de SIG comuns. Contudo, dado o grande número de fotos, recorreu-se ao programa PCI-Geomatica, concretamente ao módulo Orthoengine, que permite fazer este procedimento de forma semi-automática. O processo consiste na identificação manual das marcas fiduciais numa das fotos, que depois são procuradas de forma automática nas restantes fotos. A figura 1 mostra uma foto completa (1a) e a marca do canto superior direito (1b). 
(a)
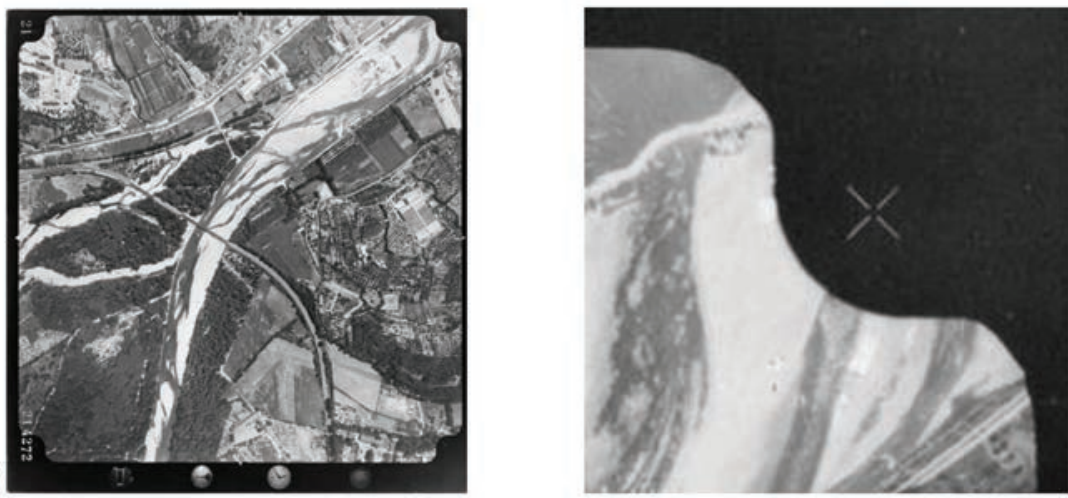

(b)

Figura 2 - Exemplo de um foto completa (a) e de uma das marcas fiduciais (b)

Este procedimento funcionou de forma correta para $63 \%$ das fotos, sendo que os erros médios quadráticos encontrados foram em geral inferiores a 1 pixel. O processo falhou nos restantes $37 \%$ das fotos, por falha na identificação de uma das marcas. A razão prende-se com o fato de nessas fotos se encontrar gravado o número da foto sobreposto a uma das marcas fiduciais. O reconhecimento automático do padrão das marcas não foi possível nesse caso. De qualquer forma em praticamente todas essas fotos a falha aconteceu apenas em uma das marcas, que facilmente foi corrigida manualmente.

Uma vez conhecidas as coordenadas imagem (linha, coluna), em unidades de pixel, foi possível fazer a retificação automática. Usou-se para isso a biblioteca de programas GDAL (Geospatial Data Abstraction Library), através dos comandos GDAL_TRANSLATE e GDALWARP. Com o primeiro fez-se a importação dos pontos de controlo para o cabeçalho da imagem. Com o segundo efetuou-se a retificação, com reamostragem bilinear, através de uma transformação afim, com tamanho de pixel fixo e seccionamento pelo centro das marcas fiduciais. Escolheu-se para resolução das imagens retificadas um pixel de $0.125 \mathrm{~mm}$, ligeiramente inferior ao da resolução das imagens $(0.127 \mathrm{~mm})$ por uma questão de multiplicidade. Os 212 $\mathrm{mm}$ de distância entre marcas fiduciais ficam exatamente cobertos por 1696 pixéis. As imagens resultantes, 1696 por 1696 pixéis, têm os cantos coincidentes com os centros das marcas fiduciais. A figura 3 mostra uma 
imagem já retificada (a) e o detalhe do canto superior esquerdo onde se pode observar parte da marca fiducial (b).

(a)
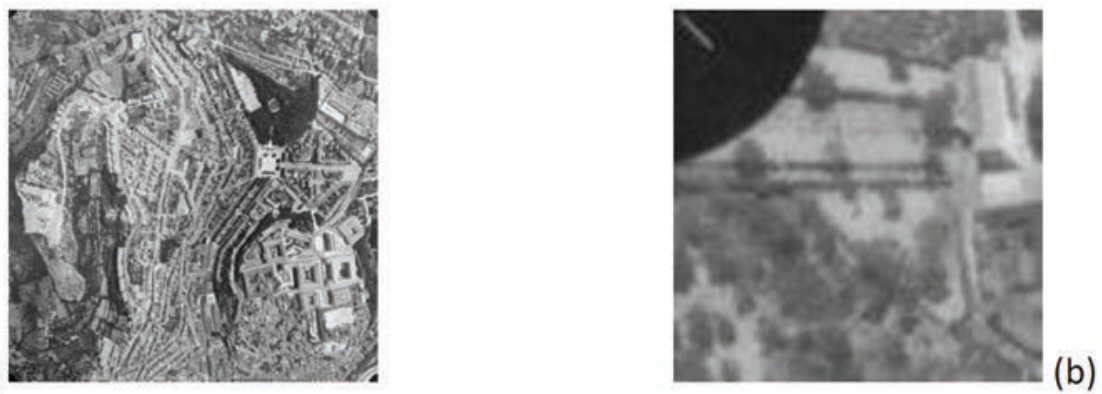

Figura 3 - Imagem retificada e ampliação do canto superior esquerdo

Na tentativa de estabelecer um paralelismo com a cadeia de processamento de imagens de VANT, tornava-se necessário um conhecimento aproximado das posições dos centros de projeção das diferentes fotos. No uso que era feito das imagens pela C. M. de Coimbra, havia sido criado um ficheiro com essas posições aproximadas, para permitir uma referência espacial mínima. A figura 4 mostra as diferentes fiadas com indicação dos pontos de início e de fim.

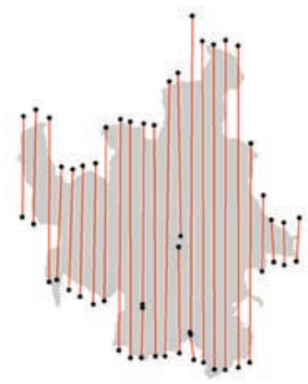

Figura 4 - Representação da localização aproximada das fiadas

As posições de todas as fotos eram conhecidas na projeção cartográfica em uso atualmente (PT-TM06). Estas coordenadas foram convertidas para geográficas, tentando reproduzir o que se passa com as imagens de VANT, que normalmente são etiquetadas com a posição dada pelo recetor GPS do sistema de navegação. A posição dos centros de projeção foi complementada pela altitude média do voo, que era de cerca de 1050 metros. 
Num passo só foram inseridos no cabeçalho de cada imagem os dados da posição aproximada do centro de projeção, da distância focal e da resolução da imagem digital. Este procedimento é feito com o programa Exiftool (HARVEY, 2014), especificando na linha de comando todas as etiquetas ("tags") a alterar, com os respetivos valores, como se descreve no exemplo seguinte

EXIFTOOL -focallength=152.19 -FocalPlaneResolutionUnit=inches

-FocalPlaneXResolution=203.2 -FocalPlaneYResolution=203.2

-GPSLatitudeRef=North -GPSLongitudeRef=West -GPSAltitude $=1040$

-GPSLatitude=40.296190 -GPSLongitude=-8.585193 R30_3871.jpg

Neste passo teve-se em consideração o facto de que a cobertura foi obtida em voos diferentes, usando diferentes lentes, concretamente duas, com as distâncias focais de $152.19 \mathrm{~mm}$ (610 fotos) e $152.63 \mathrm{~mm}$ (145 fotos). Foi tido o cuidado de inserir em cada imagem a distância focal correspondente, que se encontra gravada na foto.

Uma vez feita a inserção destes dados nos cabeçalhos das imagens estamos em condições de iniciar o processamento, utilizando o programa Agisoft Photoscan.

\subsection{Processamento}

O processamento fotogramétrico de imagens antigas apresenta uma dificuldade grande relativamente ao de fotos atuais, que é a dificuldade de encontrar pontos de controlo, devido às alterações no terreno. É comum, quando observamos uma foto de 1969 e um ortofoto atual em simultâneo, termos grande dificuldade em encontrar pontos bem definidos, que se mantenham, que possam ser usados como PF. Decidiu-se alterar a sequência de processamento apresentada no fluxograma da figura 1 , de modo a que numa fase inicial, recorrendo apenas à posição aproximada dos centros de projeção, seja gerado um orto-mosaico sem pontos de controlo. A figura 5 mostra este novo fluxograma, em que, depois dessa aproximação inicial, há a escolha de PFs e um processo iterativo de refinamento da orientação. 


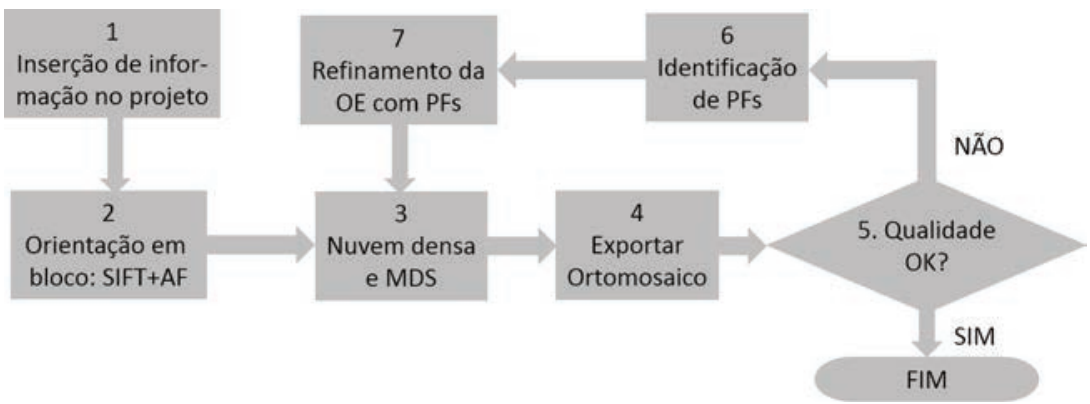

Figura 5 - Fluxograma de processamento de imagens VANT adaptado para o caso presente

As imagens foram inseridas num projeto novo, sendo lida de imediato a informação relativa à orientação interna (distância focal e tamanho do pixel) e à orientação externa (posições aproximadas dos centros de projeção). O primeiro passo a executar é a orientação automática em bloco de todas as imagens. Este processo foi executado sem problemas tendo sido concluído para todas exceto uma das imagens, que se encontrava num extremidade de uma fiada, sem mais sobreposições, e que já era fora do concelho. A figura 6 mostra um extrato da nuvem esparsa e as posições estimadas para os centros de projeção. Ao todo foram usados no processo de orientação das 754 fotos, cerca de 2.5 milhões de pontos conjugados entre as fotos.

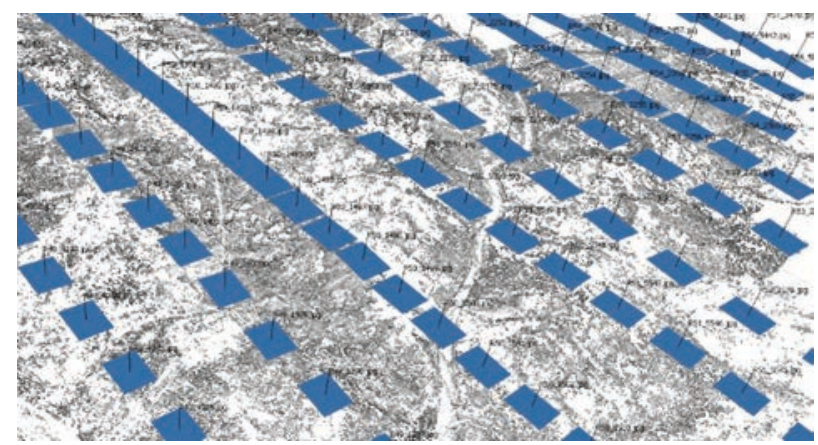

Figura 6 - Captura de ecran do programa Photoscan, mostrando a nuvem de pontos usados no alinhamento das imagens e as posições e orientações da câmara

Até agora, a única informação posicional georreferenciada fornecida foram os centros de projeção, com um baixo rigor, da ordem das dezenas ou mesmo da centena de metros. Contudo, resultam de todo o processo 
parâmetros de orientação externa que, apesar de pouco rigorosos, permitem a montagem de um orto-mosaico com georreferenciação aproximada. Este mosaico intermédio tem um interesse bastante grande no processamento porque permite agora uma mais fácil identificação de pontos de controlo por comparação com ortofotos atuais. A CMC dispõe de várias coberturas recentes de ortofotos adequadas para esta finalidade.

A figura 7 representa um extrato de 500 por 500 metros deste orto-mosaico e um ortofoto atual. Observa-se um desfasamento de um pouco mais de 40 metros. Contudo, a sobreposição das duas imagens num software de SIG permite o estabelecimento de uma correspondência entre as duas imagens, identificando pontos não alterados que podem ser usados como PF. Sem o apoio dessa georreferenciação aproximada a identificação de pontos comuns torna-se extremamente difícil.

(a)
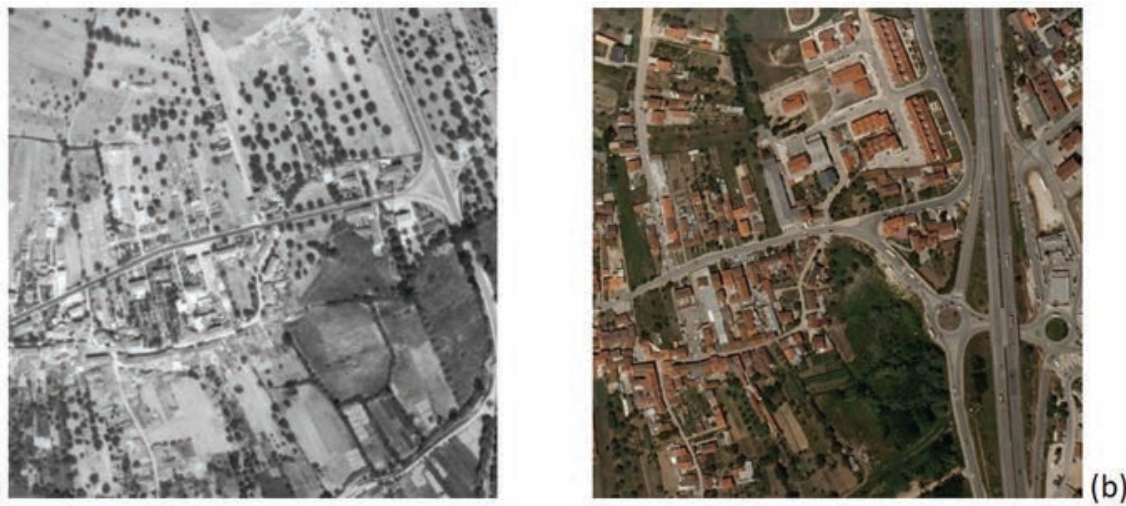

Figura 7 - Extrato do orto-mosaico aproximado (cobertura de 500 por $500 \mathrm{~m}$ ) e ortofoto atual. Observa-se um desfasamento de algumas dezenas de metros

Assim fez-se a escolha de alguns PF, preferencialmente pontos em locais com pequena variação de cota. A figura 8 mostra exemplos de dois pontos, nos ortofotos atuais e nas fotos de 1969.

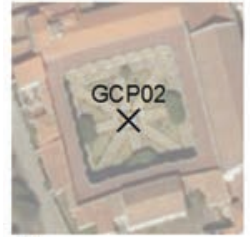

(a)

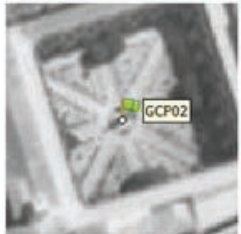

(b)

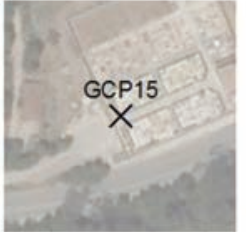

(c)

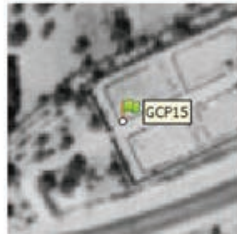

(d)

Figura 8 - Exemplos de 2 dos pontos de controlo, no ortofoto atual - (a) e (c) e identificados nas fotos de 1969 - (b) e (d) 
As coordenadas foram lidas nos ortofotos e as cotas em cartografia de escala 1:2000 que cobre partes do concelho. Nos locais onde não havia informação altimétrica e noutros onde havia dúvidas sobre a cota estimada a partir da cartografia, efetuaram-se levantamentos com GPS (dupla frequência, diferencial). Foram identificados um total de 32 pontos, distribuídos por toda a área. Foi preparada uma lista das suas coordenadas geográficas, que foi introduzida no projeto do programa Photoscan. Como existe uma georreferenciação aproximada, os pontos foram posicionados razoavelmente próximo das suas localizações corretas, em todas as imagens onde surgem, devendo o operador apenas ajustá-los manualmente, nas várias fotos onde aparecem.

O passo seguinte consiste na otimização da orientação. É recalculada a triangulação aérea com todos os pontos de controlo inseridos e todos os pontos de ligação. Nesse processo resultam resíduos que o programa fornece, e cujas estatísticas (média, erro médio quadrático, mínimo e máximo) são os indicados na Tabela 2 (valores em metros).

Os erros médios quadráticos são da ordem do metro, ou seja ligeiramente superiores à dimensão do pixel no terreno. Em casos de imagens atuais, de melhor qualidade, é normal estes erros serem inferiores à dimensão do pixel. No caso presente os pontos de controlo são em muitos casos de má qualidade e difícil identificação, tendendo a aumentar os erros. Nos pontos de ligação o erro médio quadrático nas projeções sobre as imagens foi de 0.30 pixéis.

Tabela 2 - Estatísticas dos resíduos obtidos no ajuste (média, raíz do erro médio quadrático, valores extremos)

\begin{tabular}{|c|c|c|c|}
\hline & Longitude $(\mathbf{m})$ & Latitude $(\mathbf{m})$ & Altitude $(\mathbf{m})$ \\
\hline Média & 0.06 & 0.03 & -0.18 \\
\hline REMQ & 1.06 & 0.95 & 1.00 \\
\hline Mínimo & -2.61 & -1.51 & -3.08 \\
\hline Máximo & 1.42 & 2.16 & 1.41 \\
\hline
\end{tabular}

Na componente de auto-calibração obtiveram-se correções à distância focal, à posição do ponto principal, coeficientes $\mathrm{k}_{1}, \mathrm{k}_{2}, \mathrm{k}_{3}$, do polinómio da distorção radial, e $\mathrm{p}_{1}$ e $\mathrm{p}_{2}$, da distorção tangencial. O modelo matemático aplicado é o de Brown que se encontra detalhado na documentação do programa Photoscan (AGISOFT, 2011). 
Finalmente foram exportados o modelo digital de superfície e o mosaico de ortofotos. No caso deste último a resolução considerada foi de $0.84 \mathrm{~m}$, correspondente à resolução média de todas as fotos. Dado que o MDS foi criado com resolução "média", a dimensão da grelha é o quádruplo da resolução das imagens, ou seja, de $3.36 \mathrm{~m}$.

O orto-mosaico e o MDS poderiam ser novamente analisados no SIG, podendo ser identificados mais pontos de controlo em zonas de menor qualidade. O processo poderá ser iterado até que o resultado seja globalmente satisfatório.

\section{ANÁlise Do ORTO-MOSAICO E DO MDS OBTIDOS}

A figura 9 mostra o MDS extraído e o orto-mosaico, seccionados pelo limite do concelho. O orto-mosaico tem a dimensão de cerca de 29400 por 37600 pixéis, ou seja mais de 1 giga-pixel. Foi estruturado com compressão JPEG, estruturado em tiles de 256 pixéis e com pirâmides, utilizando rotinas GDAL, sendo dessa forma facilmente manipulável em software de SIG.

De forma a avaliar o rigor altimétrico do MDS, fez-se a subtração de um MDT obtido de cartografia de escala 1:5000, tendo-se obtido diferenças com um RMS de 5.2 metros. Este valor é um majorante do erro real, dado que se está a comparar representações de entidades diferentes.

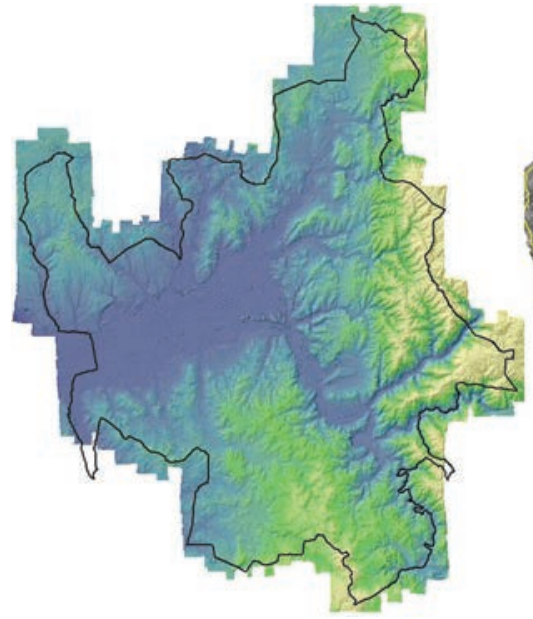

(a)

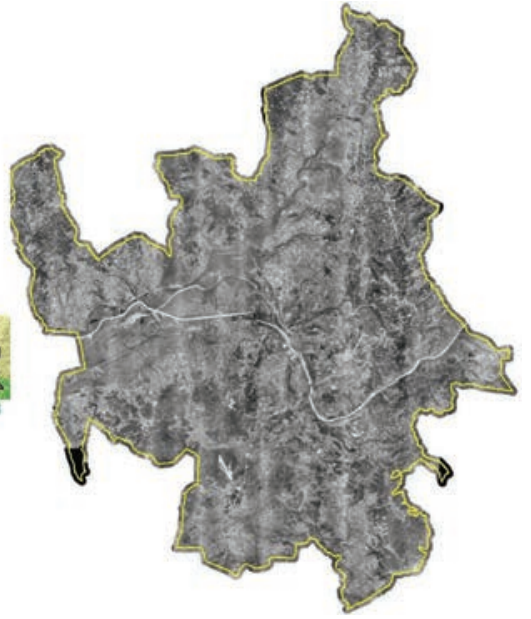

(b)

Figura 9 - Representação hipsométrica, com sombreamento, do MDS extraído (a) e orto-mosaico de todo o concelho (b) 
Relativamente ao orto-mosaico fizeram-se várias inspeções ao seu rigor posicional, de forma qualitativa, comparando-o com os ortofotos atuais e com cartografia vetorial de escalas 1:1000 e 1:2000 das zonas urbanas. Em geral há uma evidente concordância, como se pode observar na figura 10.

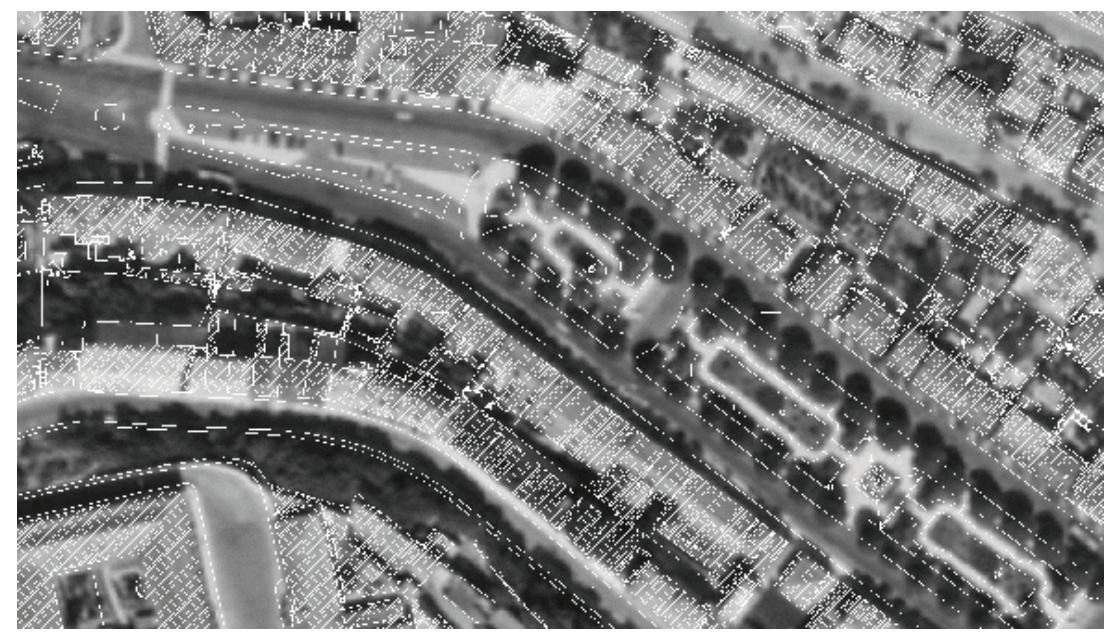

Figura 10 - Orto-mosaico com cartografia de escala 1:1000 sobreposta, num software de SIG

Procuraram-se também locais onde o orto-mosaico apresentava algumas deficiências. Algumas poderiam ser de esperar, por várias razões. Uma resulta do facto de em muitos locais a qualidade das imagens ser fraca, por exemplo por se encontrar muito clara. Nestes casos a extração do MDS pode apresentar pior qualidade, que se transmite ao ortofoto. A outra dificuldade resulta de que o ortofoto produzido é um orto verdadeiro, por fazer uso de um MDS. Dado que o MDS não consegue nas zonas urbanas um detalhe muito grande, não é possível uma adequada modelação das angulosidades dos edifícios. Facilmente se produzirão no ortofoto ondulações ou faltas de continuidade. A figura 11 mostra um exemplo desta situação. Poderia sugerir-se a ortorretificação com um MDT, por exemplo da cartografia atual. Esta abordagem não está prevista no programa Photoscan, que só permite a ortorretificação com o modelo extraído. 


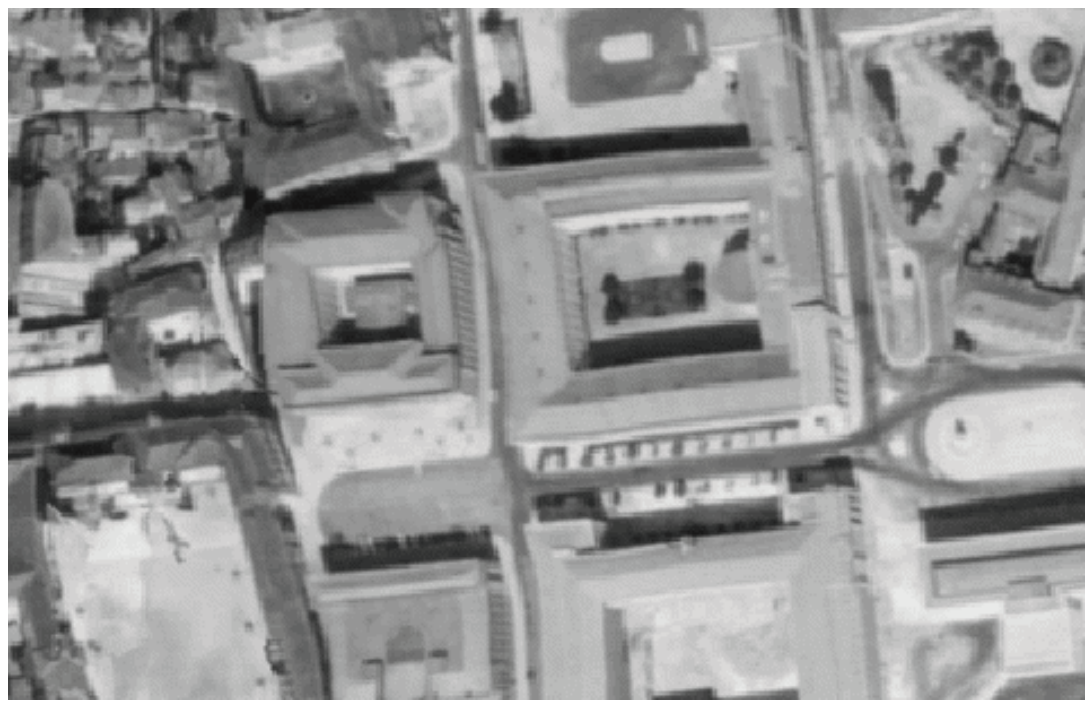

Figura 11 - Exemplo de área de falta de qualidade do orto verdadeiro

Tratando-se sobretudo de um produto para uso interno, estes problemas podem ser facilmente tolerados. Contudo poderão ocorrer situações em que seja necessário apresentar perante o cidadão extratos destas imagens. Estabeleceu-se uma estratégia de mascarar estes problemas através de edição de imagem. Tratando-se de regiões de pequena dimensão o efeito da ortorretificação não será muito crítico, podendo-se fazer uma colagem de partes da imagem original, não ortorretificada, ajustada ao orto-mosaico com uma transformação projetiva ou do tipo "rubber-sheeting". Isso foi feito na zona da Universidade de Coimbra, onde devido à altura dos edifícios, se notaram algumas imperfeições do ortofoto.

\section{CONCLUSÃo}

A metodologia descrita permitiu a criação de um orto-mosaico de forma bastante rápida e essencialmente automatizada. O processo foi algo dificultado pela qualidade das imagens, que em alguns locais era má, mas não impediu a orientação da quase totalidade das imagens. A extração da nuvem densa de pontos foi também possível, com poucas falhas. 
O mosaico produzido revelou-se muito útil, permitindo agora uma confrontação de dados atuais com o conteúdo das fotografias aéreas de 1969, por via da sobreposição em SIG de camadas corretamente georreferenciadas. $O$ rigor planimétrico não foi avaliado explicitamente com pontos de verificação, dada a dificuldade de encontrar pontos bem definidos para esse efeito. Contudo, numa avaliação qualitativa pela sobreposição com cartografia vetorial, a concordância revela-se em geral bastante boa, com desvios da ordem do metro.

Uma limitação do orto-mosaico criado é a da resolução em que foram digitalizadas as imagens (200 dpi). Teria sido possível obter mais qualidade com uma nova digitalização, por exemplo em 400 dpi, tirando mais partido do conteúdo de imagem. Contudo seria de esperar mais dificuldade com a extração automática e com a dimensão do mosaico final. Trabalho adicional a realizar será o processamento de outras coberturas disponíveis no país, nomeadamente as de 1947 e de 1958.

\section{BIBLIOGRAFIA}

AGISOFT (2011) - Agisoft Lens User Manual, Version 0.4.0.

AGISOFT (2012) - Agisoft Photoscan User Manual, Professional Edition, Version 1.0.0.

FURUKAWA, Yasutaka \& PONCE, Jean (2010) - Accurate, Dense, and Robust Multi-View Stereopsis. IEEE Transactions on Pattern Analysis and Machine Intelligence, Vol. 32, No. 8, pp 1362-1376.

EISENBEISS, Henri (2011). The Potential of Unmanned Aerial Vehicles for Mapping. Photogrammetrische Woche 2011, Dieter Fritsch (Ed.), Wichmann Verlag, Heidelberg, pp. 135-145.

GONÇALVES, José et al. (2002). Geração de ortofotos utilizando modelos digitais de superfície. Atas da III Conferência Nacional de Cartografia e Geodesia. Lidel Edições Técnicas, pp. 235-242.

HARVEY, Phill (2014) - ExifTool Home Page. http://www.sno.phy.queensu.ca/ phil/ exiftool/ (acedido em junho de 2014).

HAALA Norbert (2009) - Comeback of digital image matching. Photogrammetric Week 2009, Wichmann Verlag, Heidelberg, 289-301.

HAALA, Norbert \& ROTHERMEL, Mathias (2012) - Dense Multi-Stereo Matching for High Quality Digital Elevation Models. PFG Photogrammetrie, Fernerkundung, 
Atas das I Jornadas Lusófonas de Ciências e Tecnologias de Informação Geográfica, Sessão 10, Artigo 44

Geoinformation Jahrgang, 2012 Heft. Vol. 4 (2012), p. 331-343.

LOWE, David (2004) - Distinctive Image Features from Scale-Invariant Keypoints. International Journal of Computer Vision. Vol. 60, No. 2, pp. 91-110.

MANTA, Virgínia et al. (2011) - Planta Topográfica da cidade de Coimbra 1932/1934. Contributo para a história da cartografia obtida por fotografia aérea. Actas do IV Simpósio Luso-Brasileiro de Cartografia Histórica. Porto, Nov. 2011.

REDWEIK, Paula et al. (2010) - Triangulating the Past - Recovering. Portugal's Aerial Images Repository. Photogrammetric Engineering \& Remote Sensing, Vol. 76, No. 9. 
Série Documentos

Imprensa da Universidade de Coimbra

Coimbra University Press

2015

- U M

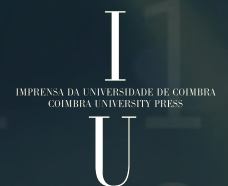

